Check for updates

Cite this: RSC Adv., 2018, 8, 15380

Received 22nd March 2018

Accepted 16th April 2018

DOI: $10.1039 / c 8 r a 02509 f$

rsc.li/rsc-advances

\title{
Cytotoxicity and ER stress-apoptosis gene expression in ZnO nanoparticle exposed THP-1 macrophages: influence of pre-incubation with BSA or palmitic acids complexed to BSA
}

\author{
Yu Gong, $\dagger^{\mathrm{a}}$ Xianqiang $\mathrm{Li}, \dagger^{\mathrm{b}}$ Guochao Liao, ${ }^{\mathrm{c}}$ Yanhuai Ding, (D) *a Juan Li (iD *a \\ and $\mathrm{Yi} \mathrm{Cao}^{\mathrm{a}}$
}

In a biological microenvironment, biological macromolecules could interact with nanoparticles (NPs) and consequently influence the toxicity of NPs. This study investigated the effects of BSA or palmitic acids complexed to BSA (PA-BSA) on the toxicity of ZnO NPs to THP-1 macrophages. Atomic force microscopy showed the increase of NP heights after pre-incubation with BSA or PA-BSA, but PA-BSA more effectively altered the hydrodynamic size and zeta potential of NPs. Pre-incubation with BSA but not PA-BSA alleviated ZnO NP induced cytotoxicity, and transmission electron microscopy confirmed fewer intrastructural changes after exposure to ZnO NPs pre-incubated with BSA. ZnO NP exposure increased intracellular $\mathrm{Zn}$ ions but decreased reactive oxygen species (ROS) and release of soluble monocyte chemotactic protein-1 (SMCP-1), whereas pre-incubation with BSA and PA-BSA induced a different pattern of intracellular $\mathrm{Zn}$ ions and modestly increased intracellular ROS. The expression of ER stress marker DDIT3 was only significantly induced after exposure to NPs pre-incubated with PA-BSA, and CASP12 expression was significantly lower after exposure to NPs pre-incubated with BSA compared to NPs with or without pre-incubation of PA-BSA. In summary, these results showed that pre-incubation with BSA was more effective compared with PA-BSA to alleviate the toxicity of ZnO NPs to THP-1 macrophages, which should be considered for the evaluation of NP toxicity in a biological microenvironment.

\section{Introduction}

When in a biological microenvironment, nanoparticles (NPs) could rapidly adsorb biological molecules because of the high free surface energy, which can consequently influence the biological effects of NPs. This suggests that it is necessary to consider the interactions between NPs and biological molecules to better evaluate the effects of NPs entering biological microenvironments. ${ }^{1}$ For example, the adsorption of serum proteins to form a protein corona has been extensively studied, and has been convincingly shown to deeply influence the stability and toxicity of NPs in circulation. ${ }^{2,3}$ Still, more studies are needed to investigate the interactions between NPs and biological

${ }^{a}$ Key Laboratory of Environment-Friendly Chemistry and Applications of Ministry Education, Laboratory of Biochemistry, College of Chemistry, Xiangtan University, Xiangtan 411105, P. R. China. E-mail: yhding@xtu.edu.cn; juanli@xtu.edu.cn

${ }^{b}$ College of Animal Science, Tarim University, Key Laboratory of Tarim Animal Husbandry Science and Technology of Xinjiang Production \& Construction Corps, Alar, 843300, P. R. China

'International Institute for Translational Chinese Medicine, Guangzhou University of Chinese Medicine, Guangzhou, Guangdong 510006, P. R. China

$\dagger$ These two authors contributed equally. molecules, especially biological macromolecules other than serum proteins. ${ }^{4}$

Recently, we and others have shown that free fatty acids could be adsorbed onto NPs, which could influence the colloidal stability and biological effects of NPs. ${ }^{5-7}$ However, under in vivo conditions a large amount of free fatty acids is complexed to proteins, but relatively few studies investigated the interactions between NPs and free fatty acids complexed to proteins. Lichtenstein et al. showed enhanced uptake of Ag NPs into Caco-2 cells if NPs were digested with the main food components, such as carbohydrates, proteins and fatty acids. ${ }^{8}$ Cao et al. showed that saturated fatty acids complexed to BSA enhanced the cytotoxicity of ZnO NPs to Caco-2 cells, which was associated with increased mitochondrial reactive oxygen species (ROS) production. ${ }^{9}$

Therefore in this study, we investigated the influences of preincubation with palmitic acids complexed to BSA (denoted as PA-BSA) on colloidal aspects and toxicity of ZnO NPs to THP-1 macrophages, and compared the effects with BSA preincubated NPs. We focused on the interactions between PA and ZnO NPs because our recent studies showed that the salt of PA could influence the colloidal aspects and toxicity of $\mathrm{ZnO}$ 
NPs, ${ }^{7,10,11}$ but the influences of biological corona formed by PABSA on the toxicity of ZnO NPs remain unknown. Atomic force microscope (AFM) was used to visualize the topographic changes of ZnO NPs due to the adsorption of BSA or PA-BSA. The changes of hydrodynamic size, zeta potential and polydispersity index (PDI) were measured to indicate the alteration of colloidal aspects of NPs. Cytotoxicity, intracellular ROS, intracellular $\mathrm{Zn}$ ions and release of soluble monocyte chemotactic protein 1 (sMCP-1) were measured in THP-1 macrophages after exposure to ZnO NPs with or without pre-incubation of BSA or PA-BSA. In addition, ultrastructural changes were also observed by transmission electron microscopy (TEM). To indicate the possible mechanisms, the expression of genes associated with ER stress and apoptosis was measured by real time RT-PCR.

\section{Materials and methods}

\section{Cell culture}

The THP-1 monocytes (ATCC) were cultured in RPMI1640 medium (Hyclone, GE Healthcare) supplemented with $10 \%$ FCS (GIBCO, South Africa) and 1\% P/S solution (Beyotime, Nantong, China) and differentiated into macrophages by using $10 \mathrm{ng}$ $\mathrm{mL}^{-1}$ phorbol 12-myristate 13-acetate (PMA; Sigma, St. Louis, MO, USA) as we previously described. ${ }^{7}$

\section{PA-BSA preparation}

PA was complexed to BSA as previously described. ${ }^{9}$ Briefly, PA (purchased from Sigma-Aldrich) was dissolved in EtOH as $200 \mathrm{mM}$ and stored at $-20{ }^{\circ} \mathrm{C}$ before use. On the day of use, PA was dissolved in $10 \% \mathrm{BSA}$ as $4 \mathrm{mM}$. After incubation at $37^{\circ} \mathrm{C}$ for about $30 \mathrm{~min}, \mathrm{PA}-\mathrm{BSA}$ was further used for experiments.

\section{ZnO NP preparation}

ZnO NPs (code XFI06; $20 \mathrm{~nm}$ ) were purchased from Nanjing XFNANO Materials Tech Co., Ltd and have been thoroughly characterized as we reported elsewhere. ${ }^{12}$ The X-ray diffractograms (XRD) indicated the hexagonal phase of NPs with an average size of $22.3 \mathrm{~nm}$. The BET surface area was measured as $19.072 \mathrm{~m}^{2} \mathrm{~g}^{-1}$. The hydrodynamic size and zeta potential of 16 $\mu \mathrm{g} \mathrm{mL}{ }^{-1}$ XFI06 suspended in water were measured as $234.4 \pm$ $3.5 \mathrm{~nm}$ and $-15.5 \pm 0.3 \mathrm{mV}$, respectively.

To make the suspension of ZnO NPs, $1.28 \mathrm{mg} \mathrm{mL}^{-1}$ NPs were suspended in MilliQ water containing $1 \mathrm{mg} \mathrm{mL}^{-1}$ BSA (SigmaAldrich, USA) and sonicated twice for $8 \mathrm{~min}$ with continuous cooling on ice by an ultrasonic processor FS-250N (20\% amplitude; Shanghai Shengxi, China). After sonication, the NPs were diluted as $40 \mu \mathrm{g} \mathrm{mL} \mathrm{m}^{-1}, 160 \mu \mathrm{g} \mathrm{mL} \mathrm{m}^{-1}$ or $640 \mu \mathrm{g} \mathrm{mL} \mathrm{m}^{-1}$ in MilliQ water, $5 \%$ BSA or $2 \mathrm{mM}$ PA-BSA. After incubated for $30 \mathrm{~min}$, the NPs were diluted ten time in serum free medium to expose cells. Control cells were exposed to equal amount of vehicles in serum free medium.

\section{In situ atomic force microscope (AFM)}

AFM was used to visualize the changes of topography of XFI06 due to the incubation with BSA or PA-BSA. Briefly, the XFI06 suspensions were prepared as $640 \mu \mathrm{g} \mathrm{mL}^{-1}$ and incubated with $5 \%$ BSA or $2 \mathrm{mM}$ FFA for at least $30 \mathrm{~min}$. After incubation, the suspensions were diluted as $64 \mu \mathrm{g} \mathrm{mL}^{-1}$ in water, $0.5 \%$ BSA or $200 \mu \mathrm{M}$ PA-PBSA. In situ AFM experiments were used to investigate the topography on a Bruker MultiMode 8 by using Peakforce mode at room temperature as we described elsewhere. A total of 50 randomly selected NPs was measured in each sample to indicate the changes of AFM heights.

\section{Hydrodynamic size and zeta potential distribution}

The stock solutions of different XFI06 were prepared as indicated above, and diluted as $64 \mu \mathrm{g} \mathrm{mL}{ }^{-1}$ in MilliQ water with or without the presence of $0.5 \%$ BSA or $200 \mu \mathrm{M}$ PA-BSA. The hydrodynamic size and zeta potential distribution as well as polydispersity index (PDI) were analyzed by using Zetasizer nano ZS90 (Malvern, Amesbury, UK). All the samples were measured in triplicate, and mean \pm SD was calculated.

\section{CCK-8 and neutral red uptake assays}

The cytotoxicity was assessed by CCK-8 (cell counting kit-8) and neutral red uptake assays by using commercial kits according to the manufacturer's instructions (Beyotime, Nantong, China). Briefly, $2.4 \times 10^{5}$ per well THP-1 macrophages were seeded in 24-well plates and exposed to $0 \mu \mathrm{g} \mathrm{mL}^{-1}, 4 \mu \mathrm{g} \mathrm{mL} \mathrm{m}^{-1}, 16 \mu \mathrm{g} \mathrm{mL}$ or $64 \mu \mathrm{g} \mathrm{mL}^{-1}$ XFI06 with or without the presence of $0.5 \%$ BSA or $200 \mu \mathrm{M}$ PA-BSA (in serum free medium). After $6 \mathrm{~h}$ exposure, the cells were rinsed once with Hanks' solution, and then the CCK-8 and neutral red uptake assays were done according to manufacturer's instructions. The products were read by an ELISA reader (Synergy HT, BioTek, Woburn, MA, USA).

\section{Transmission electron microscopy (TEM)}

TEM was used to visualize the ultrastructural changes. Briefly, THP-1 macrophages were seeded at $3 \times 10^{6}$ on $60 \mathrm{~mm}$ diameter cell culture Petri dishes. The NP suspensions were prepared as indicated above, and the cells were exposed to $64 \mu \mathrm{g} \mathrm{mL}{ }^{-1}$ XFI06 with or without the presence of $0.5 \%$ BSA or $200 \mu \mathrm{M}$ PA-BSA (in serum free medium). After $6 \mathrm{~h}$ exposure, the cells were rinsed once and then scratched by using a cell scraper. After centrifuge, the cells were fixed with $2.5 \%$ glutaraldehyde in PBS overnight, post-fixed with $1 \% \mathrm{OsO}_{4}$ for $3 \mathrm{~h}$, dehydrated in a graded series of ethanol, and embedded in epoxy resin (Epon 812). The samples were then sectioned using an ultramicrotome at $70 \mathrm{~nm}$, placed on carbon film supported by copper grids, stained with uranyl acetate and lead citrate, and observed under a TEM (JEM-1230, JEOL Ltd., Tokyo, Japan) operated at $80 \mathrm{kV}$.

\section{Intracellular ROS}

The intracellular ROS was measured by a fluorescent probe DCFH-DA as previously described. ${ }^{13}$ The fluorescent products were read at excitation $485 \pm 20 \mathrm{~nm}$ and emission $528 \pm 20 \mathrm{~nm}$ by an ELISA reader. 


\section{Intracellular $\mathrm{Zn}$ ions}

The accumulation of intracellular $\mathrm{Zn}$ ions in THP-1 macrophages was measured by using a fluorescent probe Zinquin ethyl ester (Sigma-Aldrich, Saint Louis, MO, USA), as we previously described. ${ }^{7}$

\section{ELISA}

The supernatants from CCK- 8 or neutral red uptake assays were collected and stored at $-20{ }^{\circ} \mathrm{C}$ within one month before ELISA analysis. The release of SMCP-1 was determined by an ELISA kit according to manufacturer's instruction (Neobioscience Technology Co., Ltd., Guangzhou, China). The detection limit is 7.8 pg $\mathrm{mL}^{-1}$, and the concentrations of SMCP-1 in all the samples were higher than the detection limit.

\section{Real time RT-PCR}

The mRNA level of genes associated with ER stress (HSPA5, $D D I T-3, X B P-1 s)$ and apoptosis (CASP3, CASP9, CASP12) were determined by quantitative real time RT-PCR, using GAPDH as internal control. Briefly, $1.2 \times 10^{5}$ per well THP- 1 macrophages were seeded on 6-well plates and then exposed to $0 \mu \mathrm{g} \mathrm{mL} L^{-1}$ or $64 \mu \mathrm{g} \mathrm{mL}{ }^{-1}$ XFI06 with or without the presence of $0.5 \%$ BSA or $200 \mu \mathrm{M}$ PA-BSA (in serum free medium) for $6 \mathrm{~h}$. After exposure, the cells were rinsed once and total mRNA was extracted using TRI Reagent ${ }^{\circledR}$ following manufacturer's instructions (SigmaAldrich, USA). The cDNA was synthesized by using HiFiScript cDNA Synthesis Kit following manufacturer's instructions (Cwbiotech, Beijing, China). The quantitative real-time PCR was done using UltraSYBR Mixture (Cwbiotech, Beijing, China) on PikoReal ${ }^{\text {TM }}$ qPCR system (Thermo-Fisher, USA). The primers for each gene were summarized in Table 1 . The mRNA levels were expressed as the ratio between the mRNA level of the target genes and the internal control gene using the comparative $2^{-\Delta \mathrm{Ct}}$ method.

\section{Statistics}

Unless otherwise stated, the data were expressed as mean $\pm \mathrm{SD}$ of means of 3-4 independent experiments. Two-way ANOVA followed by Tukey HSD test using $\mathrm{R}$ 3.3.3 to compare the difference; $p$ value $<0.05$ was considered to be statistically significant.

\section{Results}

\section{The topography of XFI06}

Fig. 1A shows the topography of NPs investigated by AFM, which indicated the uniform surfaces of XFI06. Pre-incubation with BSA or PA-BSA led to about 70\% increase of AFM heights of NPs (Fig. 1B).

\section{Hydrodynamic size and zeta potential distribution}

The hydrodynamic size and zeta potential distribution is shown in Fig. 2A and B, respectively. The presence of PA-BSA appeared to be more effective than BSA to shift the hydrodynamic size and zeta potential distribution of XFI06. The average hydrodynamic size of XFI06 was measured as $257.13 \pm 4.91 \mathrm{~nm}$, which was increased to $262.60 \pm 8.22 \mathrm{~nm}$ by BSA and $306.70 \pm 13.89 \mathrm{~nm}$ by PA-BSA. The average PDI of XFI06 was changed from $0.14 \pm$ 0.04 to $0.17 \pm 0.03$ by BSA and $0.32 \pm 0.04$ by PA-BSA. The average zeta potential was measured as $-13.47 \pm 0.23 \mathrm{mV}$, which was not significantly changed by BSA $(-13.00 \pm 0.46 \mathrm{mV})$ but decreased to $-19.63 \pm 1.07 \mathrm{mV}$ by PA-BSA.

\section{Cytotoxicity}

As shown in Fig. 3A, significantly decreased CCK-8 viability was observed in THP-1 macrophages after exposure to $16 \mu \mathrm{g} \mathrm{mL}$ XFI06 $(p<0.05), 64 \mu \mathrm{g} \mathrm{mL}{ }^{-1}$ XFI06 $(p<0.01), 64 \mu \mathrm{g} \mathrm{mL}{ }^{-1}$ XFI06 + BSA $(p<0.01), 16 \mu \mathrm{g} \mathrm{mL}{ }^{-1}$ XFI06 + PA-BSA $(p<0.05)$, or $64 \mu \mathrm{g}$ $\mathrm{mL}^{-1}$ XFI06 + PA-BSA $(p<0.01)$. The pre-incubation with BSA or PA-BSA did not significantly the toxicity of XFI06 as assessed by CCK-8 assay, but pre-incubation with BSA led to a relatively higher CCK- 8 viability after XFI06 exposure $(p=0.056)$. For neutral red uptake assay (Fig. 3B), significantly decreased neutral red uptake was observed following exposure to $16 \mu \mathrm{g}$ $\mathrm{mL}^{-1}$ XFI06 $(p<0.05), 64 \mu \mathrm{g} \mathrm{mL}{ }^{-1}$ XFI06 $(p<0.01), 64 \mu \mathrm{g} \mathrm{mL} L^{-1}$ XFI06 + BSA $(p<0.01), 16 \mu \mathrm{g} \mathrm{mL}{ }^{-1}$ XFI06 + PA-BSA $(p<0.01)$, or $64 \mu \mathrm{g} \mathrm{mL} \mathrm{m}^{-1}$ XFI06 + PA-BSA $(p<0.01)$. The pre-incubation with BSA $(p<0.01)$, but not PA-BSA $(p>0.05)$, significantly increased neutral red uptake in THP-1 macrophages after XFI06 exposure.

\section{Ultrastructural changes of THP-1 macrophages}

TEM was used to indicate the ultrastructural changes of THP-1 macrophages and representative images are shown in Fig. 4. THP-1 macrophages after exposure to XFI06 became relatively dark and showed intracellular vacuolation (Fig. 4A). In contrast,

Table 1 The forward (F-) and reverse (R-) primers used in this study

\begin{tabular}{|c|c|c|c|}
\hline Gene names & F-primer & R-primer & Product length \\
\hline$G A P D H$ & ACAGCCTCAAGATCATCAGC & GGTCATGAGTCCTTCCACGAT & $104 \mathrm{bp}$ \\
\hline DDIT3 & GGAAACAGAGTGGTCATTCCC & GGAAACAGAGTGGTCATTCCC & 116 bp \\
\hline$X B P-1 s$ & CCGCAGCAGGTGCAGG & GAGTCAATACCGCCAGAATCCA & $70 \mathrm{bp}$ \\
\hline HSPA5 & GAATTCCTCCTGCTCCTCGT & CAGCATCATTAACCATCCTTTCG & $180 \mathrm{bp}$ \\
\hline CASP3 & TTTGAGCCTGAGCAGAGACA & GGCAGCATCATCCACACATA & $118 \mathrm{bp}$ \\
\hline CASP9 & ACAGGCAAGCAGCAAAGTTGTCGA & AGCACCGACATCACCAAATCCTCC & 149 bp \\
\hline CASP12 & GCTGCCCACCATTGAAAGACT & ATAGCAGATTCATAGACACCAT & 119 bp \\
\hline
\end{tabular}


A

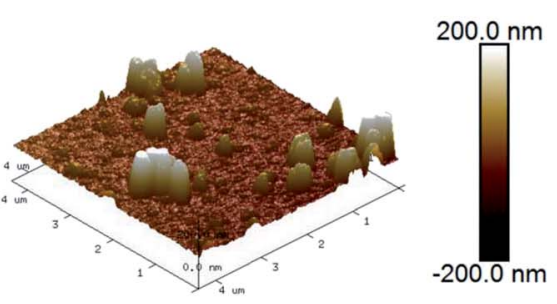

Height

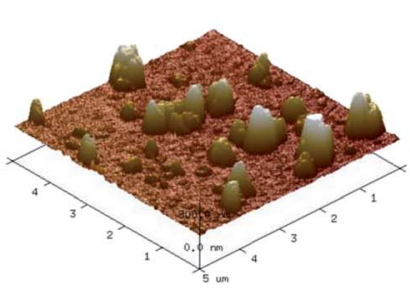

Height
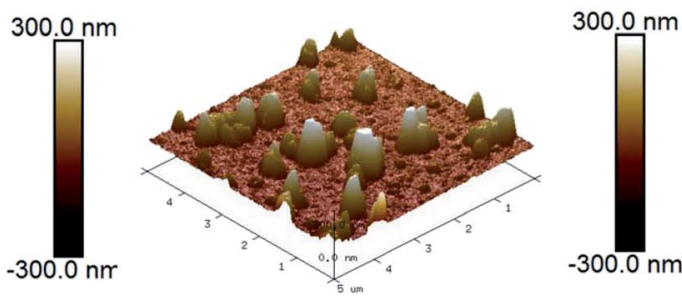

Height

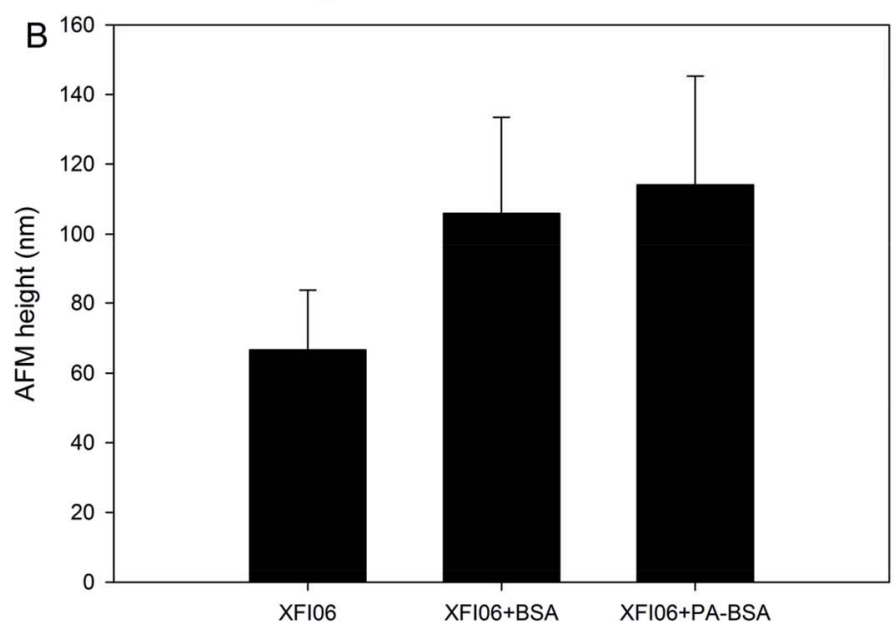

Fig. 1 The topography of ZnO NPs (code XFI06) as investigated by AFM. (A) Representative images showing XFI06 (left panel), XFI06 preincubated with BSA (middle panel) and XFI06 pre-incubated with PA-BSA (right panel). (B) The changes of AFM height of XFI06 after preincubation with BSA or PA-BSA. Data represent mean \pm SD of 50 randomly selected NPs.

the cells exposed to XFI06 pre-incubated with BSA (Fig. 4B) or PA-BSA (Fig. 4C) were whiter but still exhibited membrane breakage and intracellular vacuolation. The membrane breakage and vacuolation appeared to be more obvious for cells after exposure to XFI06 pre-incubated with PA-BSA compared with cells exposed to XFI06 pre-incubated with BSA.

\section{Intracellular $\mathrm{Zn}$ ions}

Fig. 5 depicts intracellular Zn ions after exposure to XFI06 with or without pre-incubation of BSA or PA-BSA. Exposure to XFI06 increased intracellular $\mathrm{Zn}$ ions at all concentrations to different extent, whereas pre-incubation with BSA or PA-BSA changed the pattern of intracellular $\mathrm{Zn}$ ion increase. While XFI06
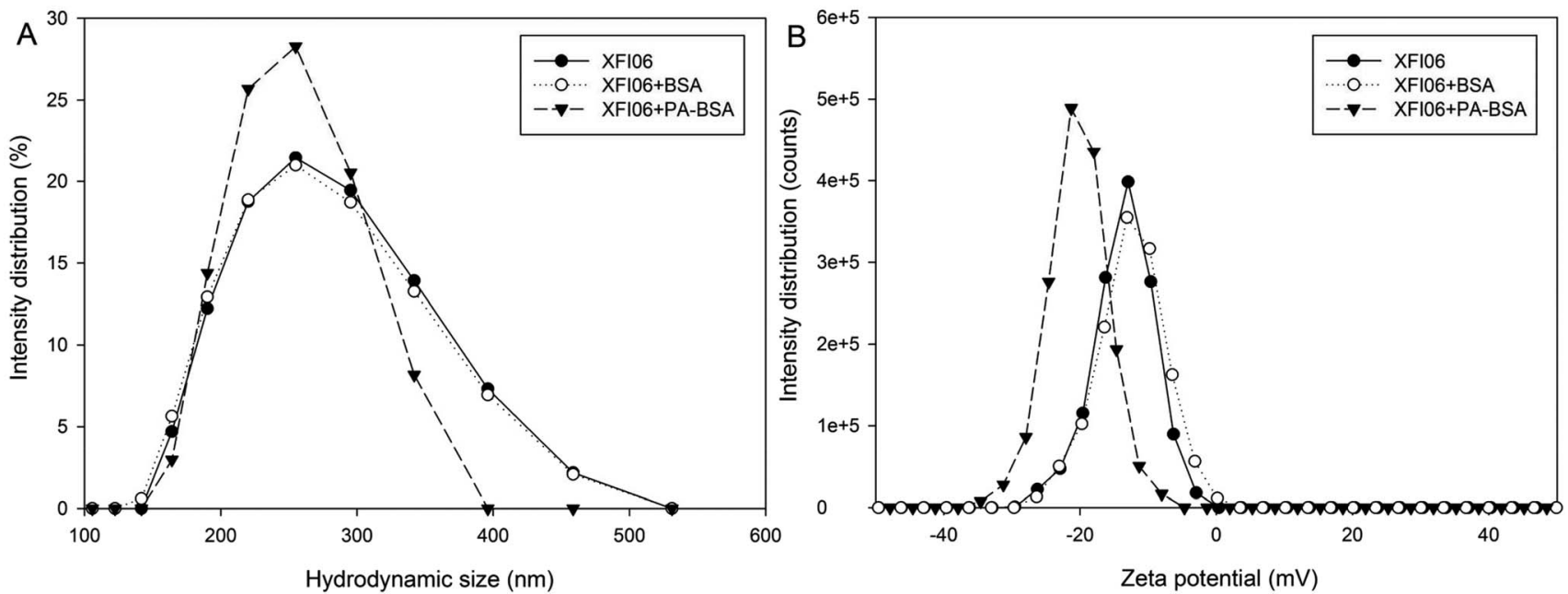

Fig. 2 The representative images showing changes of hydrodynamic size (A) and zeta potential distribution (B) of ZnO NPs (code XFI06) with or without pre-incubation of BSA or PA-BSA. 

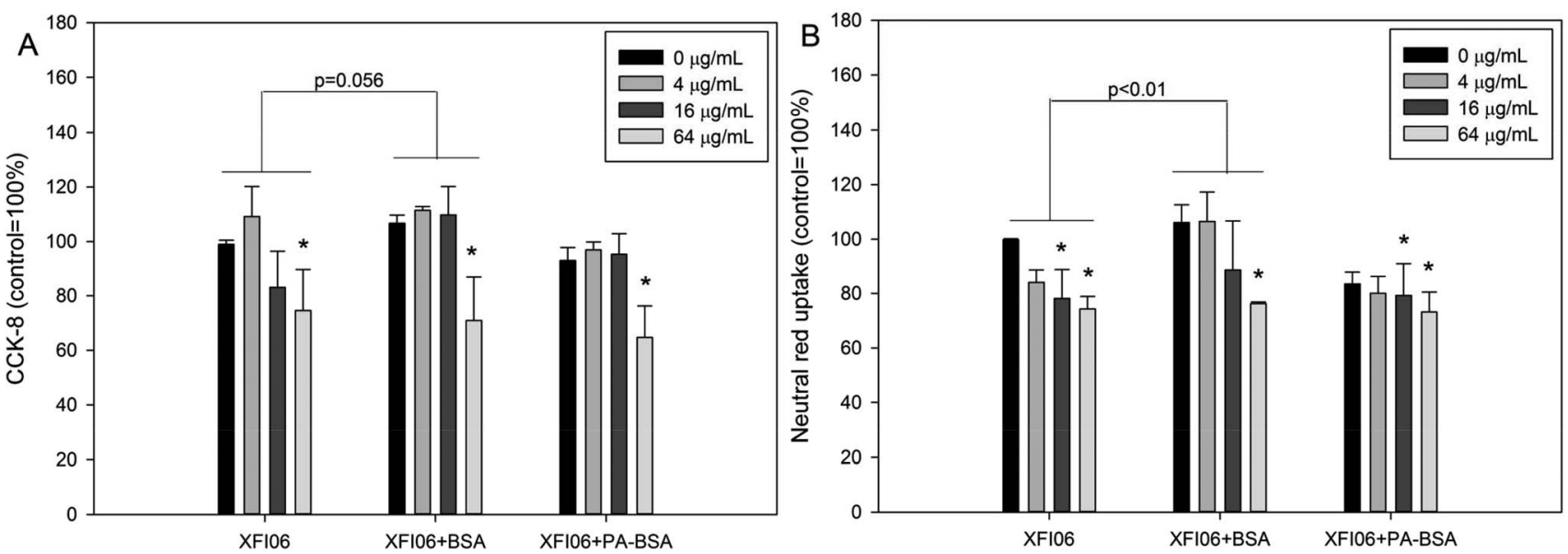

Fig. 3 Cytotoxicity of ZnO NPs (code XFI06) with or without pre-incubation with BSA or PA-BSA to THP-1 macrophages. XFI06 was preincubated with BSA or PA-BSA for $30 \mathrm{~min}$, and THP-1 macrophages were exposed to various concentrations of XFI06 with or without preincubation with BSA or PA-BSA for 6 h. CCK-8 (A) or neutral red uptake assays (B) was used to indicate the cytotoxicity. ${ }^{*} p<0.05$, compared with control.

significantly increased intracellular $\mathrm{Zn}$ ions at $4 \mu \mathrm{g} \mathrm{mL}^{-1}(p<$ 0.01 ), this concentration was shifted to $16 \mu \mathrm{g} \mathrm{mL} L^{-1}$ after preincubation with BSA or PA-BSA.

\section{Intracellular ROS}

As shown in Fig. 6, exposure to XFI06 was associated with significantly decreased intracellular ROS at all concentrations $(p$ $<0.01$ ). Pre-incubation with BSA or PA-BSA significantly increased intracellular ROS after XFI06 exposure $(p<0.01$ for both), but still lower than control.

\section{The release of SMCP-1}

The release of SMCP-1 was measured by ELISA and result is shown in Fig. 7. The release of SMCP-1 was significantly decreased after exposure to all the concentrations of XFI06 with or without pre-incubation with BSA or PA-BSA $(p<0.01)$.

\section{The expression of ER stress-apoptosis genes}

Fig. 8 shows the expression of a panel of genes associated with ER stress and apoptosis. For HSAP5 (Fig. 8A), exposure to XFI06 with or without pre-incubation of BSA or PA-BSA significantly decreased HSPA5 expression ( $p<0.01$ ). For DDIT3 (Fig. 8B), XFI06 with or without pre-incubation of BSA did not significantly affect the expression $(p>0.05)$, whereas XFI06 + PA-BSA significantly increased DDIT3 expression, which was significantly higher compared with XFI06 or XFI06 + BSA $(p<0.01)$. For XBP-1s (Fig. 8C), all the exposure groups did not significantly affect the gene expression $(p>0.05)$. For the gene expression of apoptosis genes, exposure to XFI06 with or without pre-incubation of BSA or PA-BSA did not significantly affect gene expression of CASP3 (Fig. 8D; $p>0.05$ ) but decreased that of CASP9 (Fig. 8E; $p<0.01$ ). For CASP12 (Fig. 8F), XFI06 with or without pre-incubation of BSA or PA-BSA significantly promoted the gene expression, but the expression of CASP12 was significantly lower in cells exposed to XFI06 pre-incubated with BSA compared with that in cells exposed to XFI06 $(p<0.05)$ or XFI06 + PA-BSA $(p<0.01)$.

\section{Discussion}

The aim of this study is to investigate the influences of preincubation with BSA or PA-BSA on the colloidal aspects and
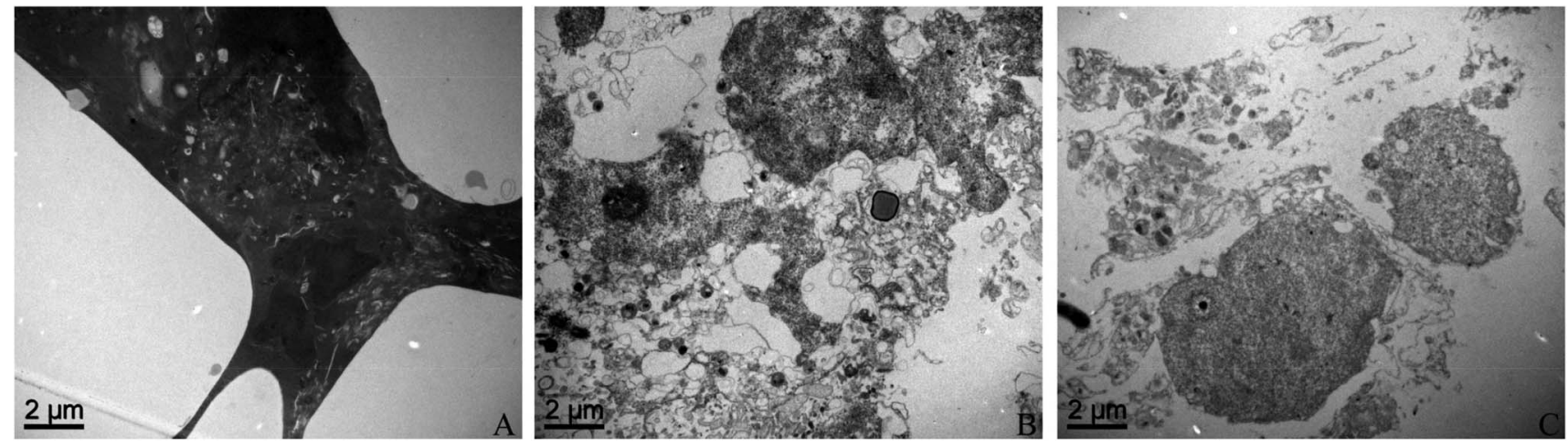

Fig. 4 Representative images showing the ultrastructural changes of THP-1 macrophages. XFI06 was pre-incubated with BSA or PA-BSA for $30 \mathrm{~min}$, and THP-1 macrophages were exposed to $64 \mu \mathrm{g} \mathrm{mL} \mathrm{L}^{-1}$ XFI06 with or without pre-incubation with BSA or PA-BSA for $6 \mathrm{~h}$. TEM was used to observe the morphology of cells after exposure to XFI06 (A), XFI06 pre-incubated with BSA (B) or XFI06 pre-incubated with PA-BSA (C). 


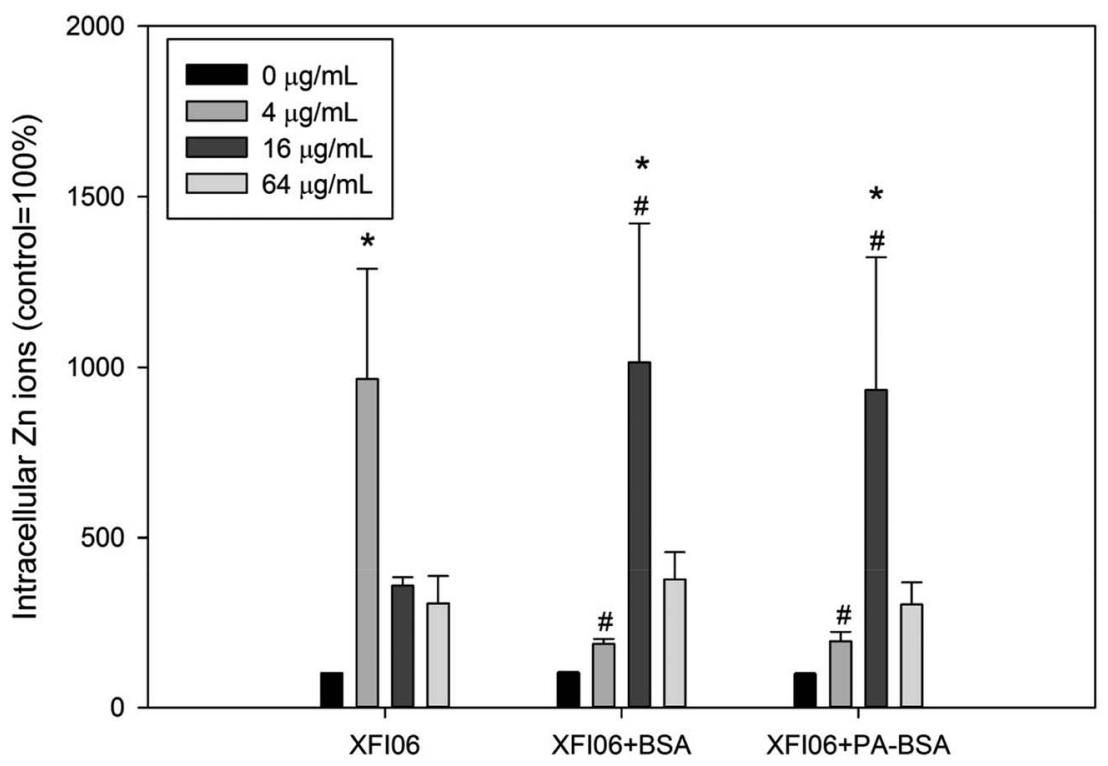

Fig. 5 The increase of intracellular Zn ions in THP-1 macrophages after exposure to ZnO NPs (code XFI06) with or without pre-incubation with BSA or PA-BSA. XFI06 was pre-incubated with BSA or PA-BSA for 30 min, and THP-1 macrophages were exposed to various concentrations of XFI06 with or without pre-incubation with BSA or PA-BSA for $6 \mathrm{~h}$. A fluorescent probe was used to measure the intracellular Zn ions. * $p<0.01$, compared with control; ${ }^{\#} p<0.01$, compared with the cells exposed to XFI06 at the same concentrations.

effects of ZnO NPs. The main findings of this study are that both BSA and PA-BSA could influence the AFM height, hydrodynamic size, zeta potential and PDI of ZnO NPs, with PA-BSA being more effective. However, BSA were more effective than PA-BSA to alleviate cytotoxicity and ultrastructural changes of
THP-1 macrophages after exposure to ZnO NPs. This could be due to that exposure to ZnO NPs pre-incubated with PA-BSA induced a relatively higher ER stress marker DDIT3 and apoptosis gene CASP12 compared with the exposure to ZnO NPs pre-incubated with BSA. Exposure to ZnO NPs increased

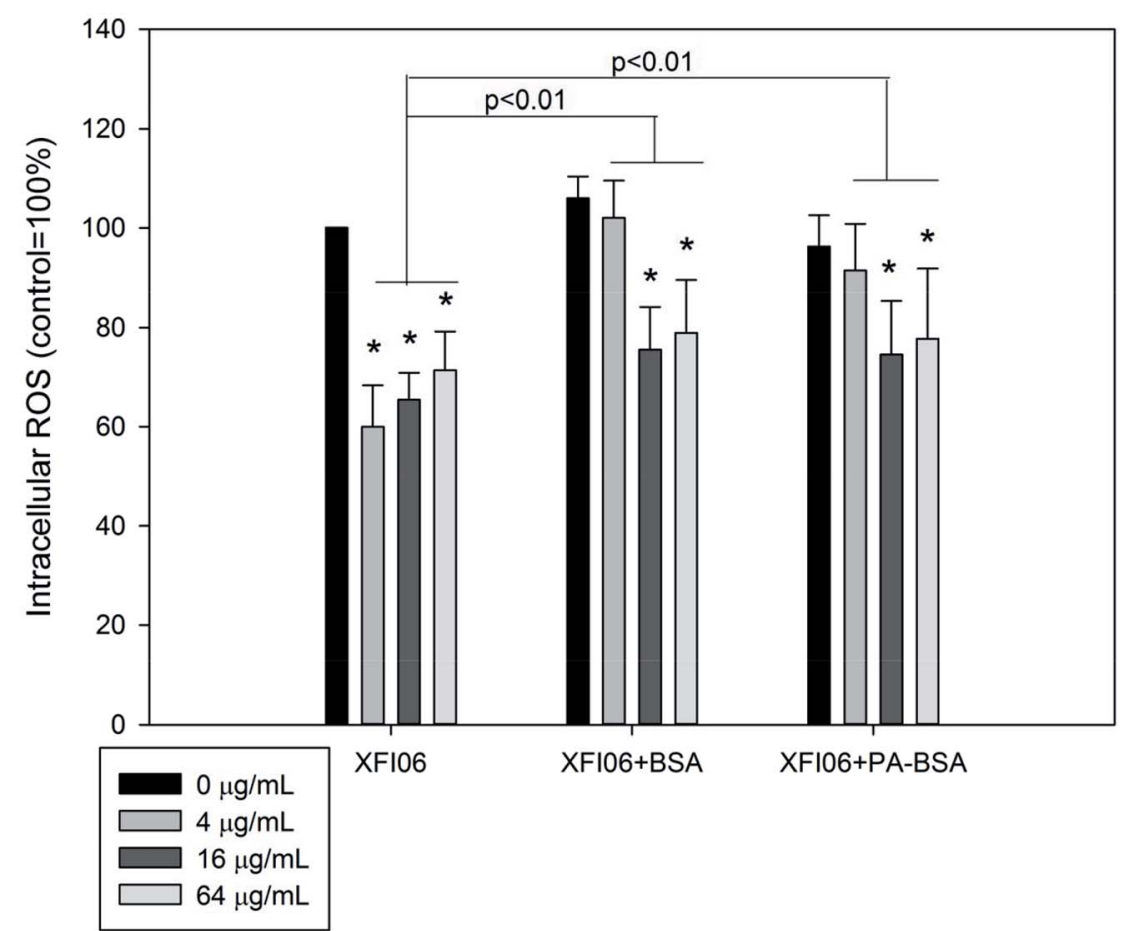

Fig. 6 The intracellular ROS in THP-1 macrophages after exposure to ZnO NPs (code XFI06) with or without pre-incubation with BSA or PA-BSA. XFI06 was pre-incubated with BSA or PA-BSA for $30 \mathrm{~min}$, and THP-1 macrophages were exposed to various concentrations of XFI06 with or without pre-incubation with BSA or PA-BSA for $6 \mathrm{~h}$. A fluorescent probe was used to measure the intracellular ROS. * $p<0.01$, compared with control. 


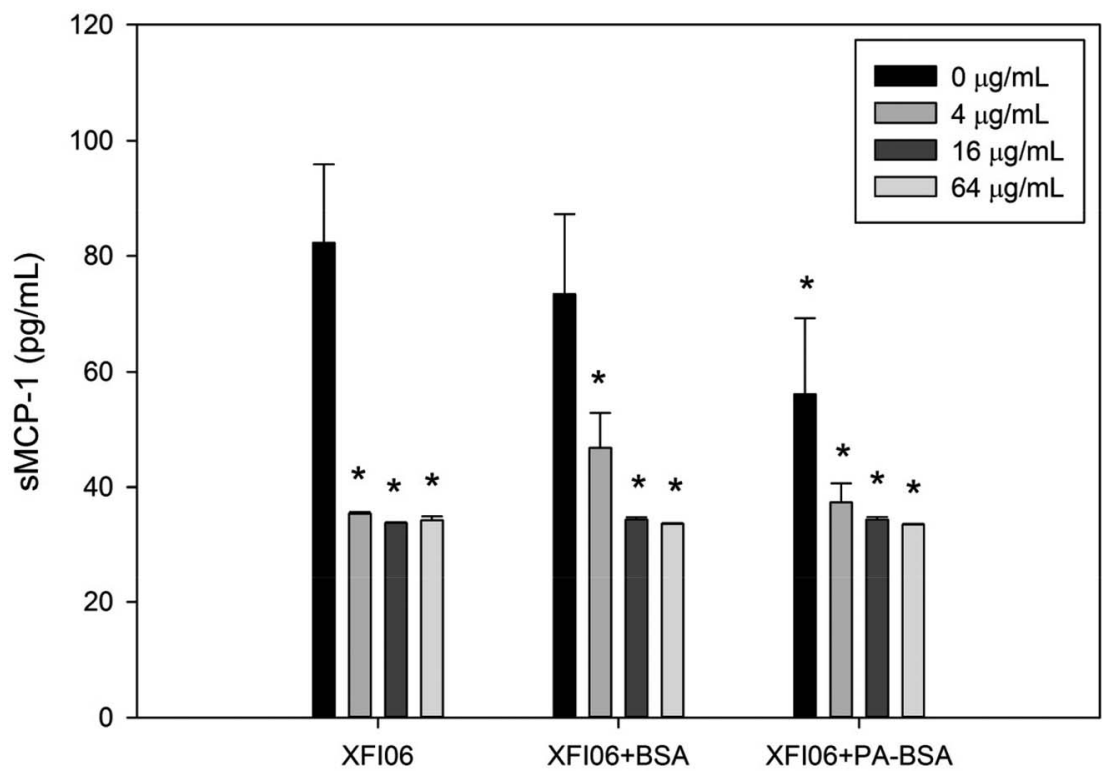

Fig. 7 The release of SMCP-1 in THP-1 macrophages after exposure to ZnO NPs (code XFI06) with or without pre-incubation with BSA or PABSA. XFI06 was pre-incubated with BSA or PA-BSA for $30 \mathrm{~min}$, and THP-1 macrophages were exposed to various concentrations of XFI06 with or without pre-incubation with BSA or PA-BSA for $6 \mathrm{~h}$. ELISA was used to measure the release of $s M C P-1$ into the supernatants. * $p<0.01$, compared with control.

intracellular Zn ions but decreased ROS and release of sMCP-1, whereas pre-incubation with BSA and PA-BSA induced a different pattern of intracellular $\mathrm{Zn}$ ions and modestly increased intracellular ROS.

We first used multiple technologies to indicate the changes of colloidal aspects of NPs due to pre-incubation with BSA or
PA-BSA, because it has been suggested that biological molecules could influence the colloidal stability and consequently the toxicity of NPs. ${ }^{14,15}$ The results from AFM showed increased AFM heights of NPs following pre-incubation with both BSA and PA-BSA (Fig. 1). To the best of knowledge, no previous study used AFM to measure the corona formation onto surface of NPs
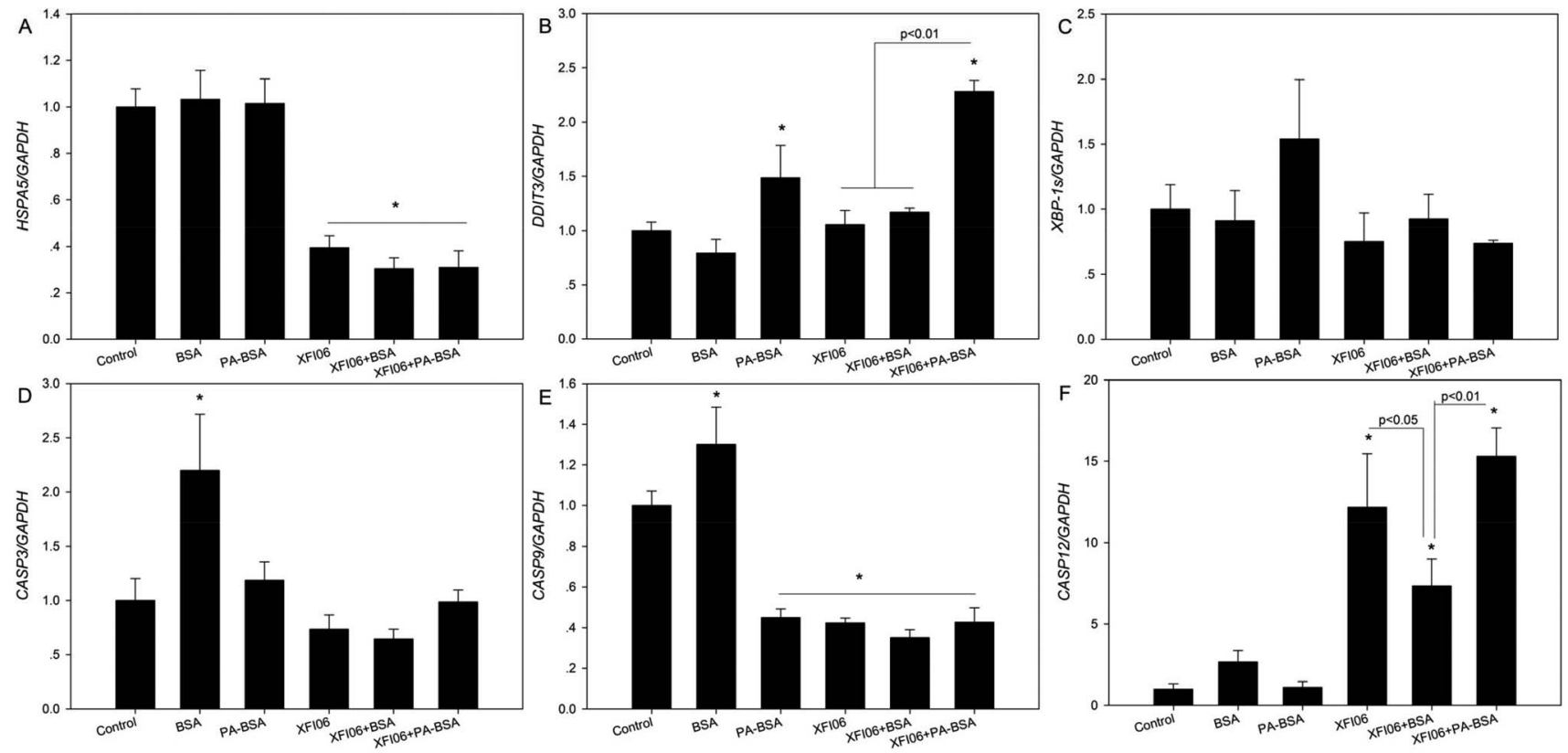

Fig. 8 The expression of genes associated with ER stress-apoptosis in THP-1 macrophages after exposure to ZnO NPs (code XFI06) with or without pre-incubation with BSA or PA-BSA. XFI06 was pre-incubated with BSA or PA-BSA for 30 min, and THP-1 macrophages were exposed to XFI06 with or without pre-incubation with BSA or PA-BSA for $6 \mathrm{~h}$. Real-time RT-PCR was used to measure the expression of HSPA5 (A), DDIT3 (B), XBP-1s (C), CASP3 (D), CASP9 (E) and CASP12 (F). *p < 0.01, compared with control. 
by fatty acids complexed to BSA, but a previous study showed size-dependent binding of NPs to phospholipids by AFM measurement. ${ }^{16}$ It is possible that NPs could adsorb lipids both complexed and not complexed to BSA. In this study we preferred to use AFM rather than TEM to measure the changes of particle sizes due to the adsorption of biological macromolecules, because AFM could allow in situ measurement of protein corona, whereas the corona might be changed during the preparation of TEM samples. ${ }^{3,17}$ Recently, we have also used in situ AFM to visualize the topographic changes of multi-walled carbon nanotubes after pre-incubation with BSA. ${ }^{18} \mathrm{AFM}$ might also be applied for the measurement of interface energy between NPs and matrix as recently shown. ${ }^{19}$ Alternatively, cryoTEM may overcome the limitations of conventional TEM to preserve protein corona. ${ }^{20,21}$ The results from this study further showed increase of hydrodynamic size and PDI as well as decrease of PDI, which was more obvious for PA-BSA compared with that of BSA (Fig. 2). Recently we also found that the salt of fatty acids could increase the hydrodynamic size and decrease zeta potential of ZnO NPs, but compared with our previous reported data, it appeared that the salt of fatty acids altered these endpoints more effectively compared with PA-BSA. ${ }^{6,7,10,11}$ Nevertheless, AFM height, hydrodynamic size, zeta potential and PDI of ZnO NPs could be changed after pre-incubation with BSA or PA-BSA, which suggested the changes of colloidal aspects of NPs due to the formation of biological protein corona.

We then investigated the cytotoxicity following exposure to ZnO NPs with or without pre-incubation of BSA or PA-BSA. The results from CCK-8 and neutral red uptake assays indicated that pre-incubation with BSA could promote cellular viability of THP-1 macrophages following ZnO NP exposure (Fig. 3). TEM study further confirmed that NPs pre-incubated with BSA induced less ultrastructural of THP-1 macrophages (Fig. 4). This is consistent with previous observations that the presence of protein corona could mitigate the toxicity of NPs. ${ }^{22,23}$ Interestingly, the results from this study also showed that PA-BSA was less effective compared with BSA to reduce the cytotoxicity of ZnO NPs. A previous study showed that PA-BSA enhanced the cytotoxicity of ZnO NPs to Caco-2 cells. ${ }^{9}$ In another study, it was shown that PA-BSA enhanced monocyte adhesion to human endothelial cells after multi-walled carbon nanotube exposure. ${ }^{24}$ We recently suggested that PA might enhance the toxic effects of NPs due to the intrinsic toxicity associated with saturated fatty acids. ${ }^{11}$ This may indicate that the influence of biological protein corona formation on toxicity of NPs is dependent on the types of biological molecules adsorbed onto NPs.

The cytotoxicity of ZnO NPs could be mediated with accumulation of intracellular $\mathrm{Zn}$ ions and/or ROS. ${ }^{25} \mathrm{In}$ this study, the results showed increased intracellular $\mathrm{Zn}$ ions (Fig. 5) but decreased ROS (Fig. 6). In our recent studies, we also showed significantly increased intracellular $\mathrm{Zn}$ ions but not intracellular ROS in a variety of cell lines following exposure to the same types of ZnO NPs, which indicated a role of intracellular Zn ions in cytotoxicity of ZnO NPs., ${ }^{611,26-28}$ However, although PA-BSA was less protective compared with BSA against ZnO NP exposure, both PA-BSA and BSA altered the pattern of increase of intracellular $\mathrm{Zn}$ ions to a similar extent. Recently we found that the salt of PA enhanced the toxicity of ZnO NPs without an influence on NP induced intracellular $\mathrm{Zn}$ ions. ${ }^{7,10}$ In addition, it has also been shown that the ER stress inducer promoted the toxicity of ZnO NPs to macrophages without further increase of intracellular $\mathrm{Zn}$ ions. ${ }^{29}$ Thus, it is possible that the toxicity of ZnO NPs could be altered by biological molecules without alterations of intracellular $\mathrm{Zn}$ ions, and the changes of cytotoxicity of ZnO NPs after pre-incubation with BSA or PA-BSA could be mediated by other mechanisms.

It has been suggested that ZnO NP exposure might modulate inflammatory responses in mammalian cells. ${ }^{30}$ To this end we determined the release of SMCP-1, and results showed that it was significantly reduced after exposure to NPs with or without pre-incubation of BSA or PA-BSA (Fig. 7). Recently we found that ZnO NP exposure significantly reduced IL-6 release from HepG2 cells $^{12}$ or A549 monolayer. ${ }^{26}$ Previous studies also showed that ZnO NPs inhibited inflammatory responses in human mast cells $^{31}$ or macrophages ${ }^{32}$ after LPS challenge. These results in combination with our data suggested that ZnO NPs might inhibit inflammatory responses. Pre-incubation with BSA or PABSA did not significantly affect the tendency of SMPC-1 release, which suggested that these biological molecules might not affect the inflammatory potential of ZnO NPs.

To explore the possible mechanisms associated with relatively lower cyto-protective effects of PA-BSA against ZnO NP exposure, we measured key regulators involved in ER stressapoptosis. ER stress is an adaptive response to accumulation of unfolded proteins which could regulate the apoptosis pathway, ${ }^{33,34}$ and induction of ER stress has been suggested to be correlated with toxicity of NPs. ${ }^{2,35}$ The results from this study showed significantly higher expression of ER stress marker DDIT3 and apoptosis gene CASP12 after exposure to NPs preincubated with PA-BSA compared with NPs with or without pre-incubation of BSA, whereas NPs with or without preincubation of BSA or PA-BSA altered other ER stressapoptosis gene expression to similar extent (Fig. 8). Since DDIT3 and CASP12 are pro-apoptosis genes that could be activated in response to prolonged ER stress, ${ }^{33-35}$ the relatively higher DDIT3 and CASP12 expression could be associated with the less cytoprotective effects of PA-BSA. Previous study showed that exposure to ZnO NPs could activate the expression of ER stress genes, ${ }^{36-38}$ here we further showed that the induction of ER stress-apoptosis gene expression is dependent on the interactions between ZnO NPs and biological molecules.

In summary, the results from this study suggested that preincubation with BSA or PA-BSA could alter the colloidal aspects of ZnO NPs, but pre-incubation with BSA was more effective than PA-BSA to alleviate ZnO NP induced cytotoxicity and ultrastructural changes of THP-1 macrophages. Exposure to $\mathrm{ZnO}$ NPs was associated with increased intracellular $\mathrm{Zn}$ ions but decreased ROS and release of SMCP-1, whereas pre-incubation with BSA and PA-BSA altered the pattern of intracellular $\mathrm{Zn}$ ions and ROS production to similar extent. Co-exposure to PABSA and ZnO NPs induced a relatively higher ER stress marker DDIT3 and apoptosis gene CASP12 compared with the exposure 
of ZnO NPs pre-incubated with BSA, which is likely associated with the changes of toxicity of ZnO NPs. The results from this study suggested complex interactions between NPs and biological macromolecules, which should be considered in future studies when assessing the toxicity of NPs in a biological microenvironment.

\section{Conflicts of interest}

No.

\section{Acknowledgements}

This work was financially supported by the National Natural Science Foundation of China (31660729).

\section{References}

1 D. Docter, D. Westmeier, M. Markiewicz, S. Stolte, S. K. Knauer and R. H. Stauber, Chem. Soc. Rev., 2015, 44, 6094.

2 Y. Cao, Y. Gong, L. Liu, Y. Zhou, X. Fang, C. Zhang, Y. Li and J. Li, J. Appl. Toxicol., 2017, 37, 1359.

3 C. Ge, J. Tian, Y. Zhao, C. Chen, R. Zhou and Z. Chai, Arch. Toxicol., 2015, 89, 519.

4 Y. Cao, J. Li, F. Liu, X. Li, Q. Jiang, S. Cheng and Y. Gu, Environ. Toxicol. Pharmacol., 2016, 46, 206.

5 M. R. Go, S. H. Bae, H. J. Kim, J. Yu and S. J. Choi, Front. Microbiol., 2017, 8, 1013.

6 X. Fang, L. Jiang, Y. Gong, J. Li, L. Liu and Y. Cao, Chem.-Biol. Interact., 2017, 278, 40.

7 Q. Jiang, X. Li, S. Cheng, Y. Gu, G. Chen, Y. Shen, Y. Xie and Y. Cao, Environ. Toxicol. Pharmacol., 2016, 48, 103.

8 D. Lichtenstein, J. Ebmeyer, P. Knappe, S. Juling, L. Bohmert, S. Selve, B. Niemann, A. Braeuning, A. F. Thunemann and A. Lampen, Biol. Chem., 2015, 396, 1255.

9 Y. Cao, M. Roursgaard, A. Kermanizadeh, S. Loft and P. Moller, Int. J. Toxicol., 2015, 34, 67.

10 Y. Gong, Y. Ji, F. Liu, J. Li and Y. Cao, J. Appl. Toxicol., 2017, 37, 895.

11 Y. Gong, L. Liu, J. Li and Y. Cao, J. Nanopart. Res., 2017, 19, 335.

12 Y. Zhou, X. Fang, Y. Gong, A. Xiao, Y. Xie, L. Liu and Y. Cao, Nanomaterials, 2017, 7, E91.

13 Y. Cao, K. Jantzen, A. C. Gouveia, A. Skovmand, M. Roursgaard, S. Loft and P. Moller, Environ. Toxicol. Pharmacol., 2015, 40, 164.

14 D. J. McClements, H. Xiao and P. Demokritou, Adv. Colloid Interface Sci., 2017, 246, 165.

15 T. L. Moore, L. Rodriguez-Lorenzo, V. Hirsch, S. Balog, D. Urban, C. Jud, B. Rothen-Rutishauser, M. Lattuada and A. Petri-Fink, Chem. Soc. Rev., 2015, 44, 6287.
16 M. V. Dwivedi, R. K. Harishchandra, O. Koshkina, M. Maskos and H. J. Galla, Biophys. J., 2014, 106, 289.

17 C. Carrillo-Carrion, M. Carril and W. J. Parak, Curr. Opin. Biotechnol., 2017, 46, 106.

18 J. Long, X. Li, Y. Kang, Y. Ding, Z. Gu and Y. Cao, RSC Adv., 2018, 8, 9253.

19 Q. Xu, J. Zhao, Y. Liu, C. Liu, J. Chen and H. Zhou, J. Nanosci. Nanotechnol., 2016, 16, 6889.

20 M. Hjort, M. Bauer, S. Gunnarsson, E. Marsell, A. A. Zakharov, G. Karlsson, E. Sanfins, C. N. Prinz, R. Wallenberg, T. Cedervall and A. Mikkelsen, Nanoscale, 2016, 8, 3936.

21 D. Klepac, H. Kostkova, S. Petrova, P. Chytil, T. Etrych, S. Kereiche, I. Raska, D. A. Weitz and S. K. Filippov, Nanoscale, 2018, 10, 6194.

22 N. Duran, C. P. Silveira, M. Duran and D. S. Martinez, J. Nanobiotechnol., 2015, 13, 55.

23 C. Corbo, R. Molinaro, A. Parodi, N. E. Toledano Furman, F. Salvatore and E. Tasciotti, Nanomedicine, 2016, 11, 81.

24 Y. Cao, M. Roursgaard, N. R. Jacobsen, P. Moller and S. Loft, Nanotoxicology, 2016, 10, 235.

25 J. Liu, X. Feng, L. Wei, L. Chen, B. Song and L. Shao, Crit. Rev. Toxicol., 2016, 46, 348.

26 T. He, J. Long, J. Li, L. Liu and Y. Cao, Environ. Toxicol. Pharmacol., 2017, 56, 233.

27 Y. Li, C. Zhang, L. Liu, Y. Gong, Y. Xie and Y. Cao, Crit. Rev. Toxicol., 2018, 28, 167.

28 C. Zhang, Y. Li, L. Liu, Y. Gong, Y. Xie and Y. Cao, J. Agric. Food Chem., 2018, 66, 1714.

29 G. Chen, Y. Shen, X. Li, Q. Jiang, S. Cheng, Y. Gu, L. Liu and Y. Cao, Environ. Toxicol. Pharmacol., 2017, 50, 103.

30 S. R. Saptarshi, A. Duschl and A. L. Lopata, Nanomedicine, 2015, 10, 2075.

31 M. H. Kim, J. H. Seo, H. M. Kim and H. J. Jeong, Eur. J. Pharmacol., 2014, 738, 31.

32 M. H. Kim and H. J. Jeong, J. Nanosci. Nanotechnol., 2015, 15, 6509.

33 C. Hetz, Nat. Rev. Mol. Cell Biol., 2012, 13, 89.

34 S. E. Logue, P. Cleary, S. Saveljeva and A. Samali, Apoptosis, 2013, 18, 537.

35 Y. Cao, J. Long, L. Liu, T. He, L. Jiang, C. Zhao and Z. Li, Life Sci., 2017, 186, 33.

36 X. Yang, H. Shao, W. Liu, W. Gu, X. Shu, Y. Mo, X. Chen, Q. Zhang and M. Jiang, Toxicol. Lett., 2015, 234, 40.

37 R. Chen, L. Huo, X. Shi, R. Bai, Z. Zhang, Y. Zhao, Y. Chang and C. Chen, ACS Nano, 2014, 8, 2562.

38 H. Kuang, P. Yang, L. Yang, Z. P. Aguilar and H. Xu, J. Hazard. Mater., 2016, 317, 119. 